
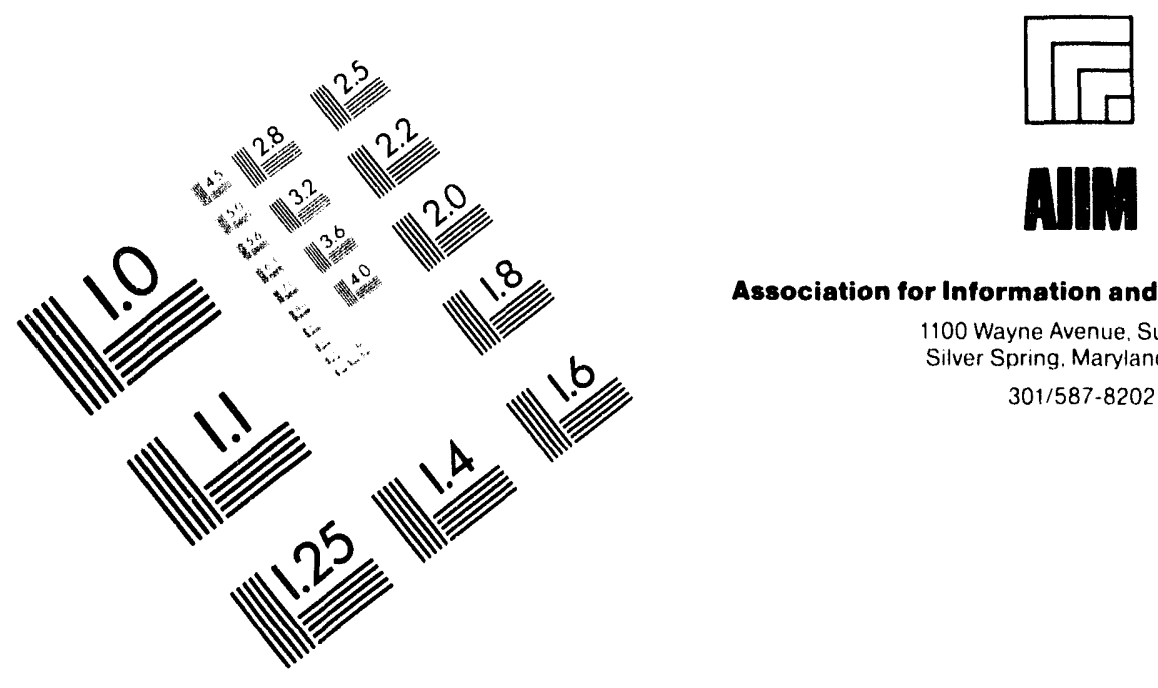

Association for Information and Image Management

1100 Wayne Avenue. Suite 1100

Silver Spring. Maryland 20910

301/587-8202

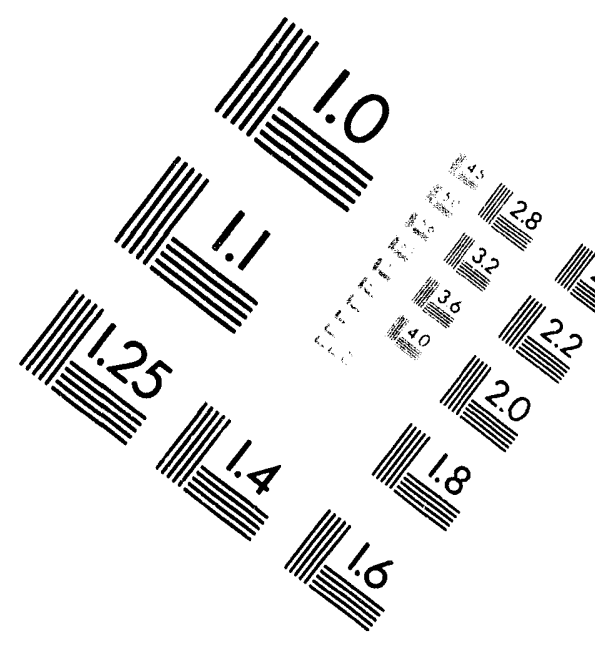

Centimeter

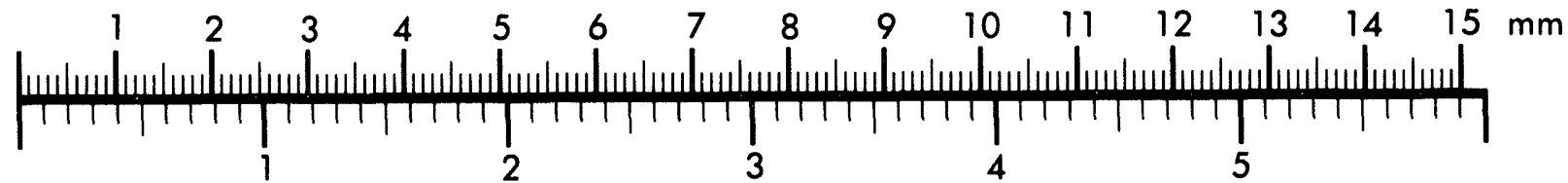

Inches
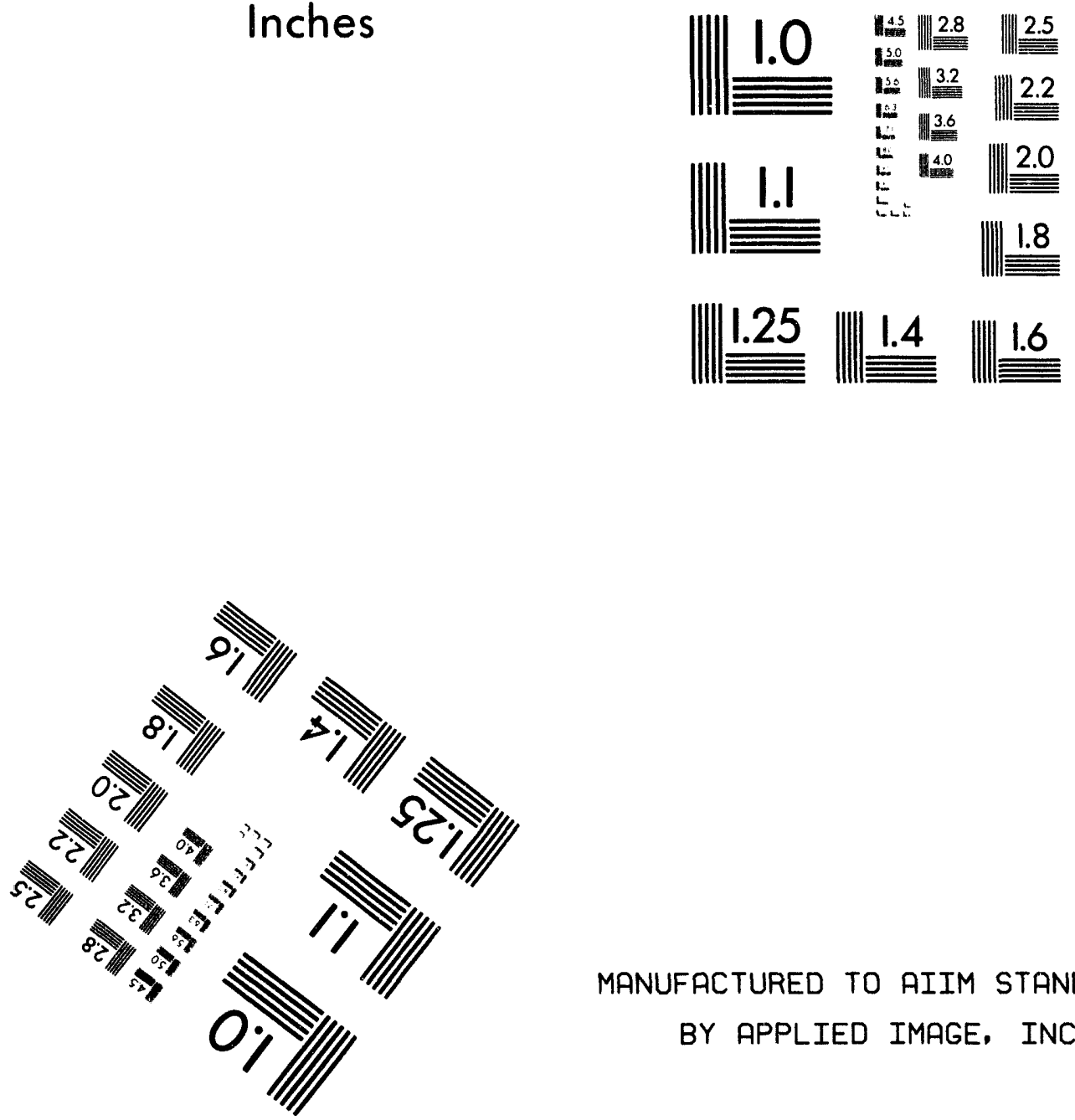

MANUFACTURED TO AIIM STANDARDS

BY APPLIED IMAGE, INC.

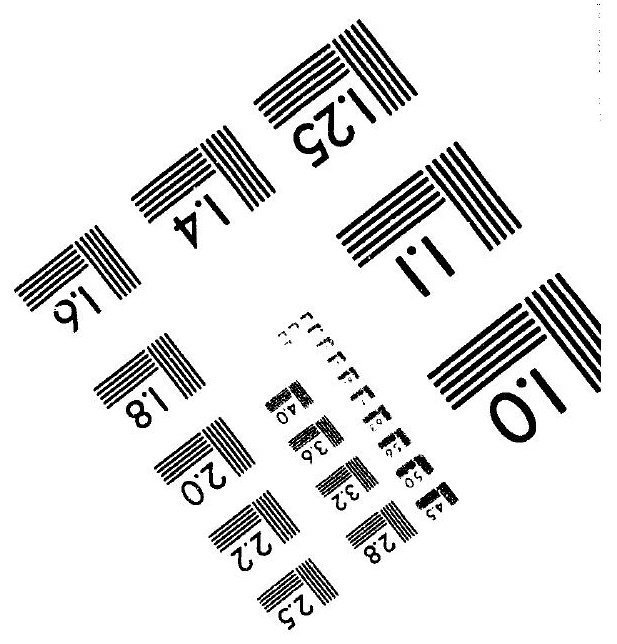



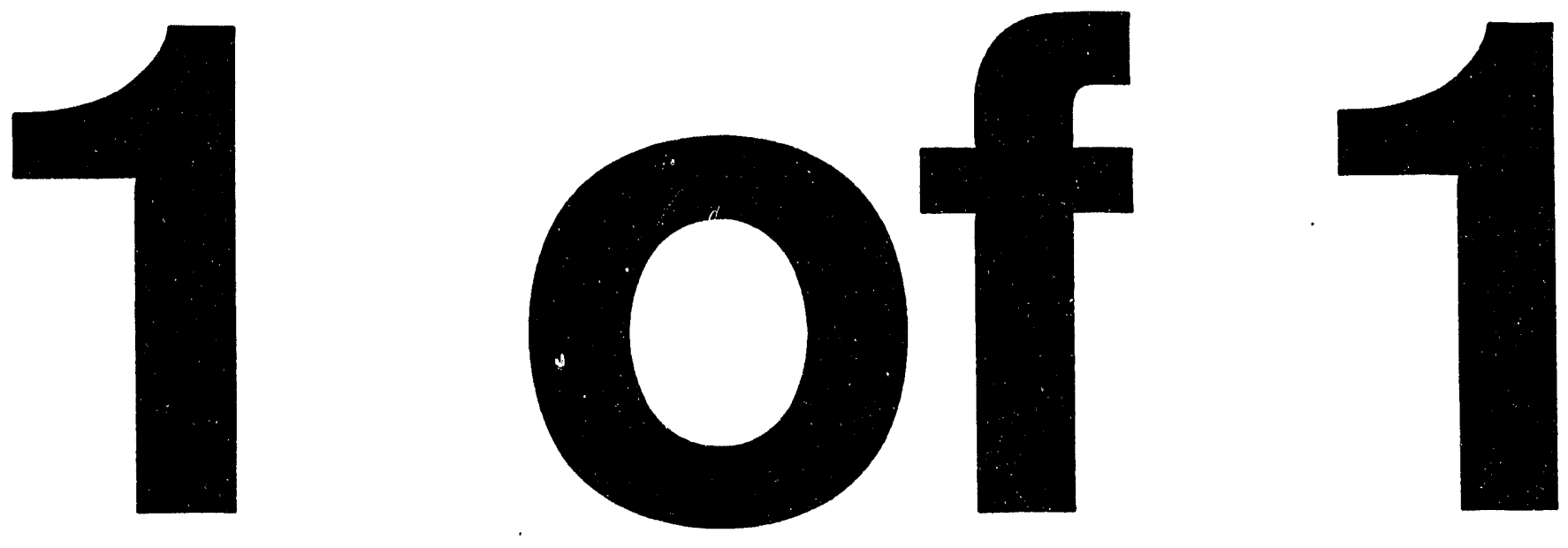
Title:

COMPUTER-DETERMINED ASSAY TIME BASED

ON PRESET PRECISION

Author(s):

Lynn A. Foster

Roland C. Hagan

E. Ray Martin

Joseph R. Wachter

Charles A. Bonner

Jack E. Malcom

Submitted to:

The Institute of Nuclear Materials Management (INMM) July 17-20, 1994

Naples, Florida

\section{DISCLAIMER}

This report was prepared as an account of work sponsored by an agency of the United States Government. Neither the United States Government nor any agency thereof, nor any of their employees, makes any warranty, express or implied, or assumes any legal liability or responsibility for the accuracy, completeness, or usefulness of any information, apparatus, product, or process disclosed, or represents that its use would not infringe privately owned rights. Reference herein to any specific commercial product, process, or service by trade name, trademark, manufacturer, or otherwise does not necessarily constitute or imply its endorsement, recommendation, or favoring by the United States Government or any agency thereof. The views and opinions of authors expressed herein do not necessarily state or reflect those of the United States Government or any ager cy thereof.
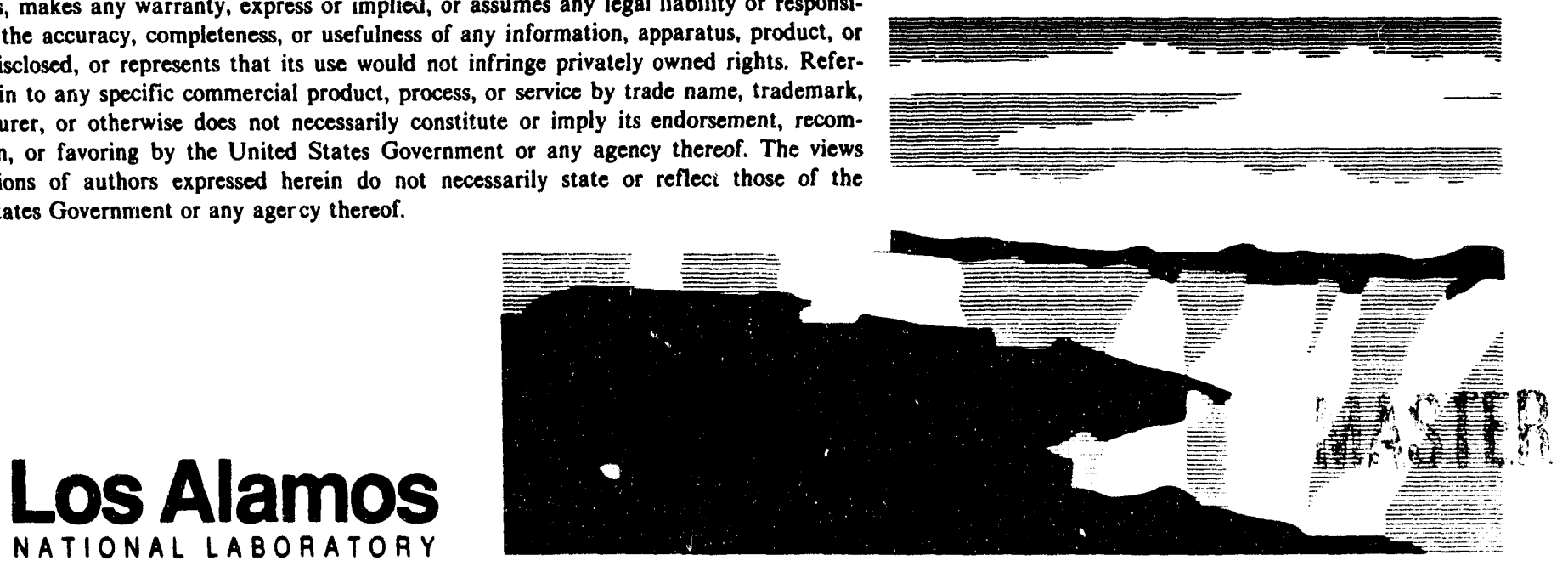

Los Alamos National Laboraton, an aftirmative action/equal oppontunity employer, is operated by the University of California for the U.S. Department of Energy under contract W.7405-ENG-36. By acceptance of this anticle, the publisher recognizes that the U.S. Government retains a nonexciusive, royalty-tree license to publish or reproduce the published form of this contribution, or to allow others to do so, for U.S. Government purposes. The Los Alamos National Laboratory requests that the publisher identity this anticle as work performed under the auspices of the U.S. Department of Energy.

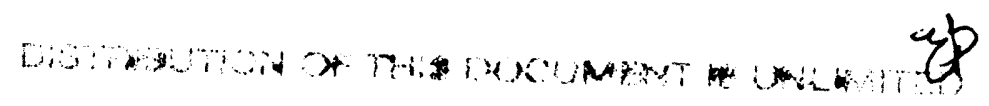




\title{
Computer-Determined Assay Time Based On Preset Precision
}

\author{
Lynn A. Foster, Roland Hagan, E. Ray Martin, Joseph R. Wachter, \\ Charles A. Bonner and Jack E. Malcom \\ Nuclear Materials Measurement and Accountability, Group NMT-4 \\ Los Alamos National Laboratory, Los Alamos, NM, USA
}

\begin{abstract}
Most current assay systems for special nuclear materials (SNM) operate on the principle of a fi:ed assay time which provides acceptable measurement precision without sacrificing the required throughput of the instrument. Waste items to be assayed for SNM content can contain a wide range of nuclear material. Counting all items for the same preset assay time results in a wide range of measurement precision and wastes time at the upper end of the calibration range. A short time sample taken at the beginning of the assay could optimize the analysis time on the basis of the required measurement precision. To illustrate the technique of automatically determining the assay time, measurements were made with a segmented gamma scanner at the Plutonium Facility of Los Alamos National Laboratory with the assay time for each segment determined by counting statistics in that segment. Segments with very little SNM were quickly determined to be below the lower limit of the measurement range and the measurement was stopped. Segments with significant SNM were optimally assayed to the preset precision. With this method the total assay time for each item is determined by the desired preset precision. This report describes the precision-based algorithm and presents the results of measurements made to test its validity.
\end{abstract}




\section{INTRODUCTION}

Nondestructive assay by segmented gamma scanner (SGS) analysis has been used successfully to measure the fissile content in radioactive scrap and waste for more than 15 years $(1,2)$. The measurement prirciple for the technique uses collimator-defined segmented analyses to relate the passive gamma-ray signal from radioactive nuclei to the quantity of fissile material contained in lowdensity matrices. The signal is corrected to compensate for matrix attenuation, rate loss, and container transmission effects. In the past, most SGS analyses have used fixed counting times for each segment in the assay. Advantages of this approach include predictable measurement durations and simplified software development. However, there are limitations to this approach. First, items cannot be measured to a preset level of precision. This is often useful because SGS operators typically require greater precision for calibration and measurement control analyses than for assays of waste and lean residues. Instruments with fixed counting times must be reset to measure for longer intervals in these instances; however, a trial measurement is needed beforehand to estimate the required time. Second, environmental and safety concerns are increasingly driving regulations that will require nondestructive analyses to be performed to fixed-precision criterion. Finally, many SGS algorithms are based on the use of a radioactive source for transmission corrections. As these sources decay, the assay time for the transmission measurement must be adjusted to maintain the same precision in the transmission correction.

To surmount these limitations, a new algorithm has been developed to enable SGS operators to specify desired precision levels. Briefly, the algorithm first determines whether any radioactive material exists in a given segment by inspecting the gamma-ray signal for a short period of time. If radioactive material is found, the algorithm determines the length of time needed to perform an assay 
of the segment to the desired precision level and instructs the instrument to assay that segment for the appropriate length of time. If no radioactive material is found in the segment, the instrument immediately moves to the next segment without further analysis. After the measurements of all segments are completed, the results are combined and the overall measurement precision is calculated.

Several advantages are gained by performing SGS assays to predetermined levels of precision. Most importantly, SGS instruments can be used with throughputs tailored to the measurement requirements. That is, the operator will have the option of specifying the desired level of measurement precision consistent with the number and type of measurements (calibration, waste assay, etc.) being performed. This flexibility allows operators to calibrate and perform measurement control checks to higher levels of precision, obviates the need for trial measurements to correlate assay time with the required precision, and may allow increased throughput. Also, operators will be better prepared to comply with evolving regulations on required waste measurement precision.

This paper contains a detailed description of the new algorithm, the results of measurements on both calibration standards and process items performed to test the algorithm's capabilities, and some concluding remarks on areas where the new method could prove useful.

\section{SYSTEM MODIFICATIONS}

Two major software modifications have been made to the SGS analysis method. First, the instrument has been redesigned to operate on the basis of a fixed or desired precision rather than on a fixed analysis time. Second, an algorithm has been added to test each segment for the presence of 
special nuclear material (SNM). If the test indicates no SNM is present, the measurement advances to the next segment. These software modifications are discussed in detail below. The two algorithm modifications are treated separately for the purposes of this discussion. In practice, the result of the test for the presence of SNM could affect the flow of the precision test.

In addition to the algorithm modifications, the hardware was modified to incorporate a light sensor to automatically detect the height of the can to be measured. At the start of the analysis, the operator must identify the type of can from a selection list. The can type identifies the container transmission to be used in the analysis. The instrument automatically determines the can height and positions the can appropriately to begin the analysis, incorporating one empty segment above and below the can.

The basic algorithm employed by the SGS is the standard two-pass method that has been in use for many years. Prior to the present modifications, the analysis was limited to a fixed time on both the shutter-open and shutter-closed passes. The new measurement method is designed around the concept of a preset precision (PSP) that is defined to be the desired overall precision (one standard deviation) in the measurement result based on the counting statistics of the analysis peak, the transmission peak, and the live-time peak.

The desired precision, or PSP, is entered as a parameter in a global parameter file. Because no prior knowledge of the item to be measured is available, it is assumed that all segments will have similar counting statistics. Under this assumption, the segment precision (SP) required for each segment in order to obtain the desired overall measurement precision is given by

$\mathrm{SP}=\mathrm{PSP} \cdot \sqrt{\mathrm{N}}$ 
where $\mathrm{N}$ is the total number of segments for the item. The number of segments is determined from the segment size, $0.5 \mathrm{in}$. for this instrument, and the can height, which is determined at the start of the analysis by the light sensor mounted on the instrument. The SP calculated from Eq. 1 is used to determine the measurement time for each segment. The following discussion applies to both the transmission measurement, with the transmission source shutter open, and the assay measurement, with the shutter closed. In the transmission measurement, the precision test is applied to the transmission peak, the $400 \mathrm{keV} \mathrm{Se}-75$ gamma-ray for Pu-239 analysis. In the assay measurement, the precision test is applied to the assay peak, the $414 \mathrm{keV}$ gamma-ray for Pu-239. The only other difference in the method for the two separate passes is that in the shutter-open pass the segment precision is defined to be $\mathrm{SP} / 1.5$. This adjustment is made for the transmission peak so that the overall measurement precision is more likely to be dominated by the statistics of the assay peak. For a four-peak assay, in which there are two transmission peaks, the precision test is applied to the lower energy transmission peak. The assumption is that the lower energy peak will be more highly attenuated and will have the poorer statistics of the two peaks.

The peak area and the variance in the peak area are calculated using the region-of-interest (ROI) summation method with a linear background subtraction(3). The assumption of symmetric placement in the background is used. The following equations are used to find the peak area, $\mathrm{A}$, and the variance in the peak area, $\mathrm{S}^{2}(\mathrm{~A})$,

$$
A=P-\frac{N_{p}}{2} \cdot\left(\frac{B_{1}}{N_{1}}+\frac{B_{2}}{N_{2}}\right)
$$


$S^{2}(A)=P+\left(\frac{N_{p}}{2 N_{1}}\right)^{2} B_{1}+\left(\frac{N_{p}}{2 N_{2}}\right)^{2} B_{2}$,

where $\mathrm{P}$ is the integral of the peak $\mathrm{ROI} ; \mathrm{B}_{1}$ and $\mathrm{B}_{2}$ are the integrals of the low- and high-background ROIs; and $\mathrm{N}_{\mathrm{p}}, \mathrm{N}_{1}$, and $\mathrm{N}_{2}$ are the number of channels in the peak and background ROIs.

The instrument first measures a segment for the test time, $T_{t}$, which is entered as a parameter in the global parameter file. The peak area and variance are calculated from Eqs. 1 and 2. The count time required to obtain the desired segment precision for that pass is calculated using

$T_{s p}=\frac{S^{2}(A) T_{t}}{(S P \cdot A)^{2}}$

One of the following three conditions will then exist.

1) $T_{s p} \leq T_{t}$

In this case, the desired segment precision was reached during the test inspection. The data collected during the test time is used for that segment and no further analysis is required. 
2) $T_{1}<T_{s p} \leq T_{\max }$

Now the calculated time to reach the segment precision is greater than the test time, meaning the desired precision was not reached during the test measurement, and the calculated time is less than the maximum count time, $T_{\max }$. The segment is then measured for an additional $T_{s p}-T_{1}$ seconds, and the new data is added to the data collected during the test measurement. The total assay time for the current pass on the segment is $T_{\mathrm{sp}}$ seconds.

3) $T_{s p}>T_{\max }$

In this case, the time required to reach the desired segment precision is greater than the maximum count time allowed for any given segment. The count rate is so low that the desired precision cannot be reached in the allowed count time. Data is collected for an additional $T_{\max }-T_{t}$ seconds, and the new data is added to the data collected during the test measurement. The total assay time for the current pass on the segment is $T_{\max }$ seconds.

If the segment precision cannot be reached within the allowed count time, case 3 above, the number of segments used in Eq. 1 to calculate SP is decremented by one. The assumption is that since the desired segment precision was not reached for that segment, it is necessary to adjust the required segment precision for the remaining segments in order to achieve the desired PSP for the overall measurement. Essentially, this is an attempt to compensate for the poor statistics on this segment by improving the precision on all other segments. The actual number of segments used in the SGS analysis does not change.

The remainder of the analysis remains the same as the normal SGS analysis. All calculations are performed on a rate basis, so the variability in the count time for each segment does nut alter the 
calculations. Basically, the corrected counts are added for each segment to determine the total corrected counts for the assay, and the standard deviations of the corrected counts are summed in quadrature to give the total measurement error. A linear calibration is applied to relate the total corrected counts to grams of SNM.

The second feature that has been added to the algorithm is an attempt to determine during the test time if there is sufficient SNM in the current segment to require further counting in that segment. This is accomplished by summing a background ROI during the shutter-closed portion of the straight through. The integral of the ROI from the straight through becomes the background. The background $\mathrm{ROI}$ is currently set from 95 to $135 \mathrm{keV}$. This area is sufficient to cover the $\mathrm{Kx}$-rays and the Pu-239 gamma-ray at $129 \mathrm{keV}$. The ROI location is stored in a parameter file that can be modified through the operator interface. When the shutter-closed pass of a segment is measured for the test time, the same background ROI is summed. Since the test ROI is located at the low end of the energy scale, the $136 \mathrm{keV}$ gamma from the Se-75 transmission source is used to correct the ROI for the sample transmission. The transmission-corrected $\mathrm{ROI}$ integral is compared to the background measurement to determine if there is any SNM in the segment. The following test is currently being used,

$$
\frac{A}{\sqrt{R_{136}}}<B+3 \sigma_{B}
$$

where $A$ is the count rate in the background $R O I$ for the current segment, $R_{136}$ is the ratio of the 136 $\mathrm{keV}$ peak area from the current segment to the peak area from the straight through measurement, B 
is the background count rate from the straight through, and $\sigma_{B}$ is one standard deviation of $B$. If the ratio, $R_{136}$, is less than $5 \%$, the sample is considered too dense to measure the $\mathrm{K} x$-rays, and the test for zero SNM is not performed.

If the result of Eq. 5 is FALSE, the analysis continues normally and the count time for the segment is determined from the required precision as previously discussed. If the result of Eq. 5 is TRUE, meaning the transmission corrected count rate of the test ROI is less than the background plus three standard deviations in the background, the decision is made that there is a negligible amount of SNM in the segment. The assay proceeds to the next segment without further analysis of the current segment. The data for that segment is not discarded, but the count time has been limited to the test time.

\section{RESULTS}

The precision algorithm was tested using a set of four dilute Pu standards ranging from 10 to 250 $\mathrm{g}$ total $\mathrm{Pu}$. The standards contain plutonium oxide $(96.4 \% \mathrm{Pu}-239)$ blended into a diatomaceous earth matrix. Table 1 compares the measurement data using the new algorithm to previous measurements made using the fixed-time analysis. The fixed-time analysis was performed using a measurement time of $25 \mathrm{sec}$. per pass, or $50 \mathrm{sec}$. per segment, for a total measurement time of $900 \mathrm{sec}$. The variable time measurements were made with a PSP of $1.5 \%$, a test time of 2 sec., and a maximum count time of $50 \mathrm{sec}$. per pass. The maximum count time, $\mathrm{T}_{\max }$, was set to allow the lowest mass standard, $10 \mathrm{~g}$, to be counted to the required precision of $1.5 \%$. On the empty can and the $10 \mathrm{~g}$ 
standard, the PSP measurement time is longer than the fixed-time analysis as the instrument attempts to achieve the desired PSP of $1.5 \%$. However, there is a significant time savings on the higher mass standards. The final two columns compare the measurement error, defined as one standard deviation in the measurement result as calculated from the measurement statistics. The fixed-time analysis shows a large variation in the measurement error, $2.5 \%$ at the low end of the mass range to $0.59 \%$ at the high end. In the variable-time analysis, the desired goal is realized, a measurement result with a predictable precision. The data from the PSP analysis is shown in Figure 1, illustrating the total measurement time and its components, the shutter-open and shutter-closed measurement times, as a function of mass of the standard. For low-mass items, the shutter-closed measurement dominates the total measurement time, since the count rate in the assay peak is low and the transmission is very high. As the mass increases, the transmission decreases and the Pu-239 signal increases, and proportionately more time is spent on the shutter-open measurement. The method automatically optimizes the time for each pass to achieve the desired precision.

Table 2 shows the result of varying the PSP from 0.5 to $4.0 \%$ when measuring the $30 \mathrm{~g}$ standard. The estimated \%RSD is the standard deviation in the results of 10 repeated runs. The individual $\% \mathrm{RSD}$ is the average measurement error, as calculated from the peak statistics, for the same 10 runs. There is good agreement between the actual spread in the measurement data, the estimated \% RSD, and the PSP requested at the start of the analysis.

Finally, Table 3 shows the measurement results of a number of process items measured recently as part of our remeasurement program. The measurements were made with a desired PSP of $1.5 \%$, a $5 \mathrm{sec}$. test time, and a $50 \mathrm{sec}$. maximum time per pass. The data shows the breakdown of the total measurement time into the shutter-closed, SC, and shutter-open, SO, passes. The measurement error and the result in grams of total Pu are also shown. The measurement error shows a spread of 1.6 to 
$2.0 \%$, reasonably close to the desired PSP of $1.5 \%$.

The test for zero SNM has not been sufficiently completed at this time and detailed results will not be discussed. The preliminary results indicate the test is extremely sensitive. We are now working on optimizing the placement of the background ROI to achieve the desired sensitivity and still retain a reasonable probability of skipping segments that do not contain SNM. Using the current ROI, the sensitivity is such that few empty segments are skipped, due to scattering of gammas emitted from plutonium in other segments. This may be improved by moving the test ROI to a higher energy region.

\section{CONCLUSION}

The initial results of the SGS modifications described in this report look very promising. The precision algorithm has already been placed into service on one of our SGS instruments. The test for zero SNM will not be used until further testing has been completed. Implementation of the precision algorithm has solved a problem that has plagued the measurement control program for some time. When a measurement control assay is performed, the measurement passes if the final result is within a specified percentage of the nominal standard value. Due to limitations of the present system, the specified percentage is a constant for each instrument, not a function of the Pu mass as it should be for a fixed-time analysis. The percentage is normally chosen for measurements toward the upper mass range of the calibration where large measurement errors are unacceptable. Measurement control measurements made using low-mass standards often resulted in failures because of the poorer precision of the measurement result, even though nothing was wrong with the instrument. The 
measurement time could be adjusted to provide the desired precision on the low-mass standards in order to alleviate this problem. However, this would lead to unnecessarily long count times on the high-mass standards. With the PSP algorithm, any standard can be measured to a previously determined precision in the minimum amount of time necessary to complete the measurement. In our NDA lab, three measurement control checks are performed each day. Thus, it becomes important to minimize the time the instrument spends performing these daily checks.

Another problem with SGS measurements is the short half life, about 120 days, of the Se-75 source used for the transmission correction. The shutter-open measurement time is usually chosen io provide the required measurement statistics toward the end of the useful life of the source. This results in over counting the transmission measurement when the source is new. In addition to adjusting the transmission measurement for sample density, the PSP algorithm will compensate for the source decay by adjusting the measurement time appropriately toward the end of the useful life of the transmission source.

Clearly, the most serious limitation to the PSP algorithm is the assumption of a homogeneous distribution of nuclear material so all segments will have similar counting statistics. While the SGS standards, and even process items to some extent, will satisfy this assumption, it is not expected to be a reasonable assumption for waste items. This assumption could be relaxed by scanning the entire item at the beginning of the assay. The distribution of nuclear material in the item would then be known, and the algorithm could optimize the count time based on this distribution. This approach would involve some additional time to complete the assay, perhaps $2 \mathrm{~min}$. to scan a typical can. However, the benefit gained would be a much more predictable error in the final assay result. 


\section{ACKNOWLEDGMENTS}

We would like to thank the staff of the Los Alamos National Laboratory Plutonium Facility NDA laboratory for their support and help in obtaining the data presented in this report.

Reference herein to any specific commercial product, process, or service by tradename, trademark, manufacturer, or otherwise, does not necessarily constitute or imply its endorsement, recommendation, or favoring by the Regents of the University of California, the United States Government, or any agency thereof.

\section{REFERENCES}

1. T.D. R.eilly and J.L. Parker, "A Guide to Gamma-Ray Assay for Nuclear Material Accountability," Los Alamos Scientific Laboratory report LA-5794-M (March 1975).

2. E.R. Martin, D.F. Jones, and J.L. Parker, "Gamma-Ray Measurements with the Segmented Gamma Scanner," Los Alamos Scientific Laboratory report LA-7059-M (December 1977).

3. "Passive Nondestructive Assay of Nuclear Materials. Doug Reilly, Norbert Ensslin, Hastings Smith, Jr., and Sarah Kreimer Eds. (United States Nuclear Regulatory Commission, NUREG/CR5550, March 1991). 
Table 1. Comparison of fixed-time to variable-time measurements over a range of SNM from 0 to 250 grams. The specified PSP was $1.5 \%$.

\begin{tabular}{|c|c|c|c|c|}
\hline \multirow{2}{*}{ SNM Mass } & $\begin{array}{c}\text { Measurement Time } \\
\text { (seconds) }\end{array}$ & \% Time Difference* & \multicolumn{2}{|c|}{ \% Relative Standard Deviation** } \\
\cline { 4 - 5 } & 998 & +11 & Fixed Time & Variable Time \\
\hline Empty Can & 1012 & +12 & $\cdots$ & $\cdots$ \\
\hline 10 grams & 565 & -37 & 2.5 & 1.7 \\
\hline 30 grams & 307 & -66 & 0.89 & 1.6 \\
\hline 100 grams & 280 & -69 & 0.59 & 1.6 \\
\hline 250 grams & & & 1.5 \\
\hline
\end{tabular}

* Percent change in measurement time from the fixed-time analysis to the variable-time analysis. The fixed time analysis was set for 25 seconds for each pass on each segment, 18 segments per item.

** One relative standard deviation as calculated during the analysis from propagation of measurement statistics.

Table 2. Results of varying the desired \% PSP when measuring a $30 \mathrm{~g}$ dilute plutonium oxide standard.

\begin{tabular}{|c|c|c|c|c|}
\hline \multirow{2}{*}{$\%$ PSP } & \multicolumn{2}{|c|}{ Measurement Time } & \multirow{2}{*}{ Estimated \%RSD* } & Individual \%RSD** \\
\cline { 2 - 5 } & Shutter Open & Shutter Closed & & 0.49 \\
\hline 0.5 & 866 & 3607 & 1.20 & 1.10 \\
\hline 1.0 & 228 & 927 & 2.48 & 2.12 \\
\hline 2.0 & 62 & 300 & 3.25 & 3.96 \\
\hline 4.0 & 36 & 118 & & \\
\hline * Percent relative standard deviation in a set of 10 repeated runs. \\
**Average measurement error calculated during the analysis for each individual run.
\end{tabular}


Table 3. Measurement results for typical process items and residuesfrom the Plutonium Facility. Measured with a desired PSP of $1.5 \%$.

\begin{tabular}{|c|c|c|c|c|c|}
\hline \multirow{2}{*}{$\begin{array}{c}\text { Item } \\
\text { Description }\end{array}$} & \multicolumn{2}{|c|}{ Measurement Time (sec) } & \multirow{2}{*}{ \%RSD } & Pu (g) \\
\cline { 2 - 6 } & SO & SC & Total & & \\
\hline OH Cake & 178 & 693 & 871 & 1.8 & 53.0 \\
\hline OH Cake & 387 & 389 & 776 & 1.6 & 188.2 \\
\hline OH Cake & 263 & 741 & 1004 & 1.7 & 94.8 \\
\hline OH Cake & 293 & 525 & 818 & 1.7 & 79.6 \\
\hline Sweepings & 208 & 820 & 1028 & 1.9 & 54.7 \\
\hline Salt Residue & 356 & 765 & 1121 & 1.8 & 50.7 \\
\hline Salt Residue & 539 & 729 & 1268 & 2.0 & 144.1 \\
\hline Oxide Cake & 490 & 801 & 1291 & 1.7 & 69.7 \\
\hline
\end{tabular}




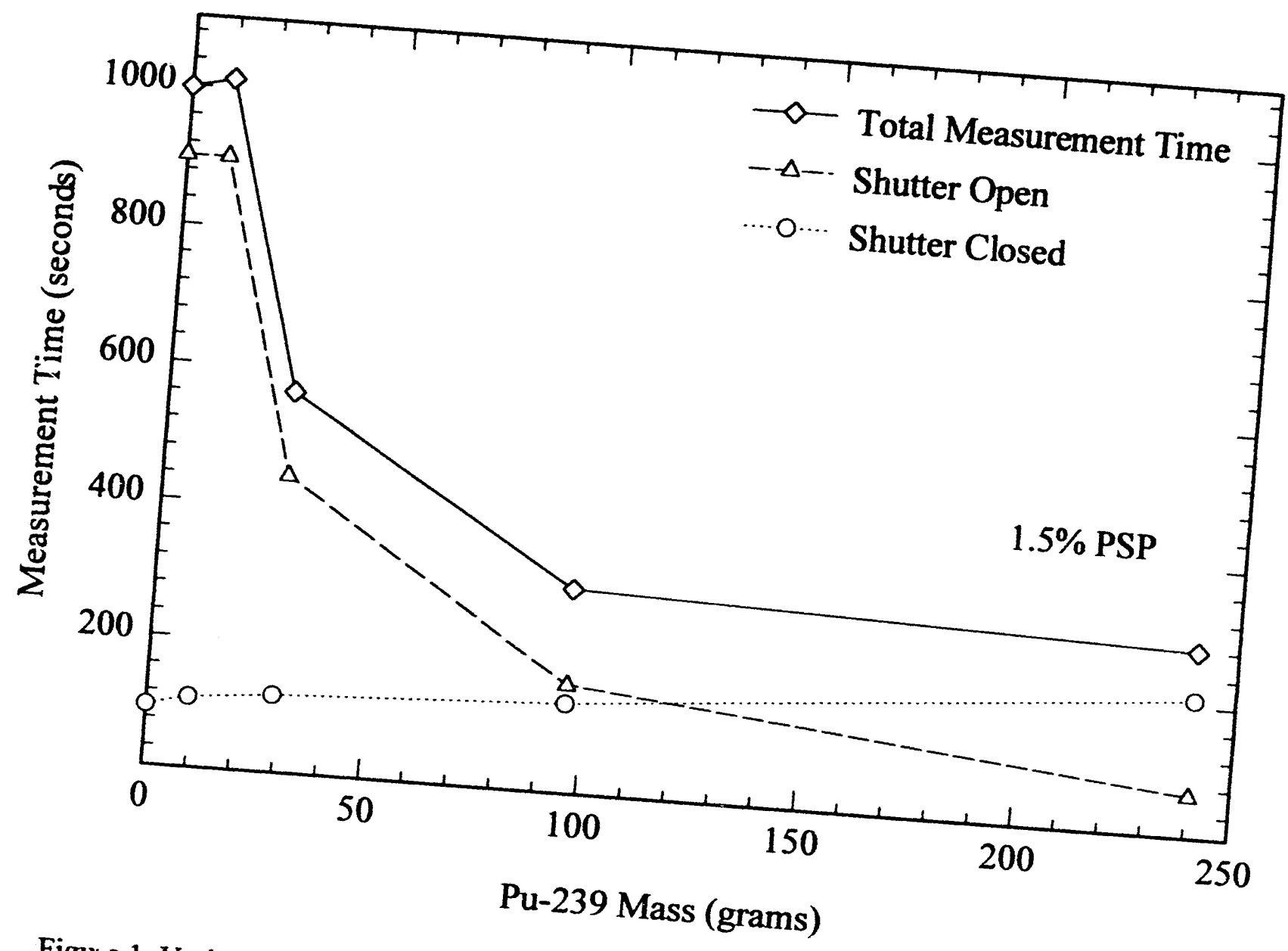

Figuie 1. Variation in SGS measurement time as a function of SNM mass using a PSP of $1.5 \%$ 

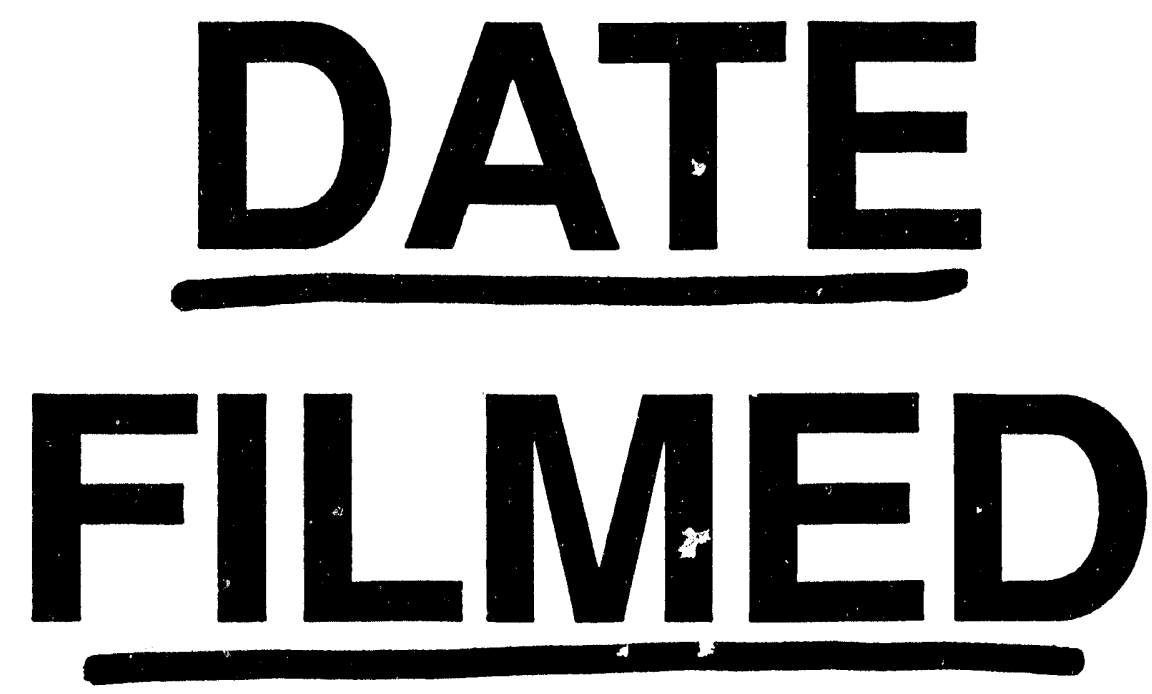

$9 / 7 / 94$
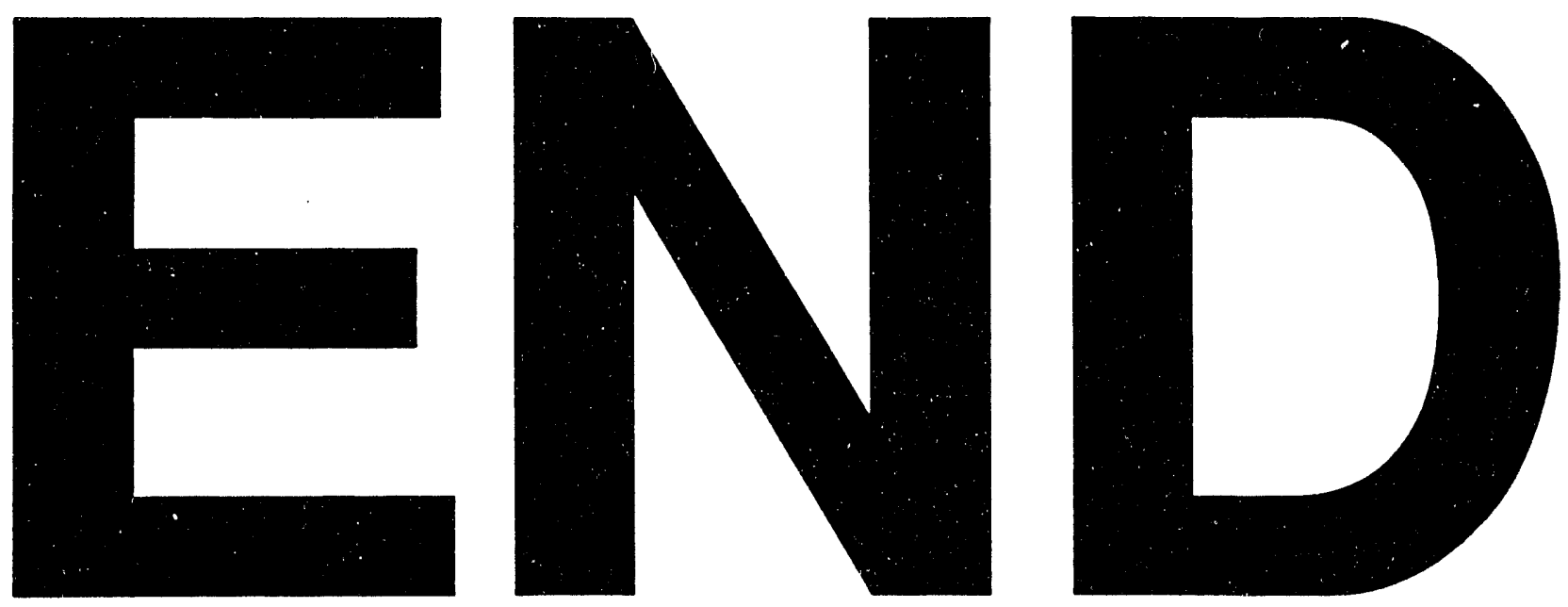


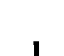

